These days, some initial studies started to address the health of workers exposed to novel types of manufactured nanomaterials. These studies face three main challenges: exposure assessment, identification of suitable effect markers and size of populations. The relatively small current workforces in individual countries will probably necessitate the pooling of cohorts internationally. However, at the moment, the necessary conditions for such a pooling are not in place: namely agreements on design, exposure and effect characterisation are not in place. To bridge this gap and to provide a coherent approach in view of future epidemiological research, we recently proposed a roadmap [1] to reach global consensus on need a well defined, globally harmonised framework for the careful choice of materials, exposure characterisation, identification of study populations, definition of health endpoints, evaluation of appropriateness of study designs, data collection and analysis, and interpretation of the results. The proposed strategy should ensure that the costs of action are not disproportionate to the potential benefits, and importantly, that the approach is pragmatic and practical. Moreover, we should aim to go beyond the collection of health complaints, illness statistics or even counts of deaths: the manifestation of such clear endpoints would indicate a failure of preventive measures. Instead, we should agree on a minimum set of biomarkers and metrics of early effects for acute and chronic diseases while evaluating how concepts of systems biology, gene activation and epigenetics can inform such studies on outcomes and related biomarkers of potential interest.

\section{MONITORING AND MODELLING OF EXPOSURE TO MANUFACTURED NANO OBJECTS, AGGLOMERATES \& AGGREGATES (NOAA) FOR EPIDEMIOLOGIC STUDIES}

D H Brouwer, Fransman, Pronk, Tielemans. TNO, Zeist, Nederland

\subsection{6/oemed-2013-101717.187}

Introduction A major challenge for setting up human field studies is the identification of sufficiently large number of workers with exposure to NOAAs. Pooling of data is necessary but requires harmonisation of methods. A multi-metric exposure approach is proposed, which may be better correlated to health effects, however, an appropriate exposure estimate has not been developed yet. Currently, workers' activities/tasks related to NOAA are time wise highly variable resulting in considerable within-worker, between-day variances. No validated models exist to predict exposure to NOAA, however, the concepts of such a source-receptor model have been presented, and for specific activities (i.e. powder handling or spraying) more detailed models are being developed. Meanwhile larger datasets on (mostly task-based) occupational (estimates of) exposure to NOAA are available, e.g. the NANOSH study, or will be built. Database structures are actively developed, e.g. the PEROSH -NECID database, and harmonised workplace exposure studies generating data will populate the NECID database in near future.

Methods and Results The challenges for exposure assessment for epidemiologic studies can be addressed by developing taskbased exposure matrices profiles covering scenario's across the product chain of NOAA. Building blocks for such task-based exposure matrices will be existing models and those that are under development, e.g. Stoffenmanager nano, NanoSafer, further analysis of existing datasets to demonstrate task-specific exposures and tailored task-based measurements. Combined with worker specific information on type of NOAA, and frequency, duration etc. of activities/tasks per job title, estimates for exposure can be derived. In addition, co-exposures from process or combustion derived ultrafine particles will be taken into account. A multi-pathway approach is used, however, the focus will be on the inhalation route. Currently, the feasibility of such an approach is explored in a pilot study, which is supported by an extensive measurement campaign in the facility.

\section{FROM THE VERY SMALL TO THE VERY LARGE: CHALLENGES IN CONDUCTING EPIDEMIOLOGIC STUDIES OF US WORKERS EXPOSED TO CARBON NANOTUBES}

K Schubauer-Berigan, Dahm, Deddens, Birch, Evans, Erdely. National Institute for Occupational Safety and Health, Cincinnati, United States of America

\subsection{6/oemed-2013-101717.188}

Presentation Time: 11:00 - 11:30

Objectives Carbon nanotubes (CNT) and carbon nanofibers (CNF) are among the first nanomaterials to reach commercial use in the US and are also showing evidence of serious health effects at occupationally relevant levels in toxicology studies. The objective of our work was to design an epidemiologic study of early possible health effects among US workers exposed to CNT and CNF, taking into account small workforce sizes, a global manufacturing and distribution system, uncertainty about which exposure metrics may best correlate with health effects, and short available latency.

Methods Initial exposure characterisation was conducted at 15 US manufacturers and users of CNT and CNF to determine the most specific and useful exposure metrics. Possible markers of early pulmonary, cardiovascular, and malignant health effects were identified from animal toxicology studies and epidemiologic research among populations exposed to ambient ultrafine particles. Power analyses were conducted to determine appropriate sample sizes.

Results A cross-sectional exposure assessment and epidemiology study was designed; it will include 100 workers from at least 10 US facilities making or using CNT or CNF. This study, now in progress, evaluates elemental mass and electron microscopybased exposure metrics for each worker, along with early health outcomes including spirometry measures, blood pressure, and approximately 40 biomarkers of inflammation, oxidative stress, pulmonary fibrosis, cardiovascular disease, and cancer. The study will account for ambient ultrafine exposure using a combination of background sampling and non-specific direct-reading instruments that operate in the nanoscale range.

Conclusions Cross-sectional epidemiologic designs for nanomaterial exposures are feasible, but small workforce sizes and generally short latency limit power; cohort studies for outcomes such as malignant and nonmalignant respiratory and other disease may require international pooling. Researchers should collaborate to identify the most suitable exposure metrics and early health outcomes.

\section{MEDICAL SURVEILLANCE AND EPIDEMIOLOGIC STUDIES OF ENGINEERED NANOMATERIALS (ENM) WORKERS IN FRANCE}

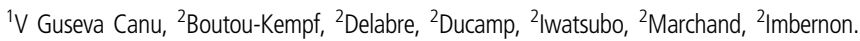
${ }^{1}$ Saint Maurice, France; ${ }^{2}$ French Institute for Public Health Surveillance (InVS), St-Maurice, France

10.1136/oemed-2013-101717.189 
Objectives To depict the present situation regarding ENM-workers medical and epidemiological surveillances in France and discuss its advancement.

Methods During 2008-2010 the InVS conducted an exploratory study based on in site visits of French facilities using/producing ENM with aim to explore the ENM exposure circumstances, ENM-workers medical surveillance and other epi-surveillance development prerequisites. This "field" information was completed with systematic scientific and statutory bibliography reviews and discussions within two working groups. One included scientists from the French Institute for Public Health Research (IReSP) and focused the epi-surveillance development aspects, while another included physicians from the Occupational medical inspection department at the Ministry of Labour and focused medical surveillance aspects.

Results Since neither epidemiologic nor individual medical surveillance existed specifically for ENM-workers despite a likelihood of exposure in some facilities, InVS developed a protocol for an integrated surveillance system of French workers potentially exposed to ENM. It consists of a multi-step methodology starting with an ENM-exposure registry. ENM-workers will be identified using a 3-level approach: 1-selection of companies concerned with ENM exposure (based on questionnaire and compulsory declaration), 2-in site exposure assessment and identification of the job/tasks with ENM exposure (based on jobexposure-matrix construction, further supplemented with measurements), and 3-registration of ENM-workers (based on inclusion criteria and additional self-questionnaire). The registration is planned for three years focusing two ENM, carbon nanotubes and nano- $\mathrm{TiO}_{2}$. The two corresponding prospective cohorts will pursue epidemiologic surveillance objectives and serve as a basis for performing cross-sectional/panel studies with specific research objectives.

Conclusion The French ENM-workers surveillance is actively developing. Companies and workers inclusion questionnaires are designed and protocol is operational for starting in early 2013, after approval from national ethical committees (still awaited). The results coming from the first six-month operation should be informative in terms of data quality, numbers of facilities and workers with ENM-exposure likelihood.

\section{Session: Mini symposium II: Occupational cancer in Europe (SHECAN)}

\section{SHECAN - METHODOLOGY FOR EXPOSURE ASSESSMENT: MAKING THE MOST OF LIMITED DATA}

${ }^{1}$ MG N Gorman Ng, 'van Tongeren, ${ }^{2}$ Hutchings, ${ }^{3}$ Mistry, ${ }^{3}$ Corden, 'Lamb, 'SanchezJimenez, 'Shafrir, ${ }^{3}$ Sobey, ${ }^{2}$ Rushton, 'Cherrie. 'Institute of Occupational Medicine, Edinburgh, United Kingdom; '2Department of Epidemiology and Biostatistics, Imperial College London, London, United Kingdom; ${ }^{3}$ AMEC, London, United Kingdom

10.1136/oemed-2013-101717.190

Introduction The prevalence and level of exposure for each assessed agent was estimated for every European member state and relevant industry. These estimates provided the basis for the heath impact assessment. Detailed exposure data were unavailable for many member states and industries. The strategies used to create these estimates using limited available data will be discussed using the estimates produced for respirable crystalline silica (RCS) as an example.
Methodology For 18 out of 25 assessed agents data from the CAREX project were used to estimate exposure prevalence. The proportion of exposed workers in each industry was averaged across countries for which data from 2000 or later were available. The average proportion was multiplied by the number of employees in the industry in each of the remaining member states in 2006 (from the Structural Business Statistics and Labour Force Survey available from EUROSTAT) to estimate the number of exposed workers. For agents that were not included in CAREX, exposure prevalence was estimated using data from trade associations and other stakeholders; from available exposure databases; or by assuming that all workers in exposed industries were exposed.

The level of exposure was assessed using data from the published scientific literature, European Risk Assessment Reports, exposure databases, and trade associations. Industries were classified as high, medium or low exposure and a representative geometric mean (GM) and geometric standard deviation (GSD) was selected for each "medium" and "high" exposure industry. The overall weighted GM and GSD for each substance was estimated across all medium/high exposed industries with Monte Carlo simulation.

Discussion Due to limited data availability, estimates were conservative in every instance. Had more data been available both the prevalence and exposure level estimates may have been lower, demonstrating the need for exposure measurement data from industry to be made available for research.

\section{SHECAN - METHODOLOGY FOR THE HEALTH IMPACT ASSESSMENT: THE STRENGTHS AND WEAKNESSES OF THIS APPROACH}

L Rushton, Hutchings. Imperial College London, London, United Kingdom

\subsection{6/oemed-2013-101717.191}

Objective To provide current estimates of occupational cancers in the EU associated with the relevant substances and future trends under different scenarios of change of exposure; these data provide the input into the socioeconomic assessment.

Methods We calculated attributable fractions together with numbers of deaths and cancer registrations, Disability Adjusted Life Years (DALYs) and Years of Life Lost using risk estimates from published literature and national data sources to estimate proportions exposed.

Results More than 1,000 attributable cancers were estimated to occur in the next 60 years for each of eleven substances if no action is taken; total estimated attributable deaths over this period were $>700,000$. Respirable crystalline silica (RCS) and diesel engine exhaust were particularly important giving an estimated 470,000 and 430,000 incident cancers between 2010 and 2069. There were only seven substances or mixtures where there was a health benefit in terms of avoided cancer cases over the 60 years from introducing an OEL giving between $0.2 \%$ and $39 \%$ reduction in deaths from the baseline estimate. The largest benefits arise from the introduction of OELs for RCS, hardwood dust, hexavalent chrome and rubber fume. The highest percentage reduction in incident cases was for the OEL for rubber fume (39\%), followed by hardwood dust at $1 \mathrm{mg} / \mathrm{m}^{3}$ (28\%) and RCS at $0.05 \mathrm{mg} / \mathrm{m}^{3}(23 \%)$.

Conclusions Assumptions made in our methodology and uncertainties and inaccuracies in the data may have introduced biases into our estimates. Potential sources of bias include 